\title{
Reporting of Adverse Events in Surgical Trials: Critical Appraisal of Current Practice
}

\author{
Rachel Rosenthal · Henry Hoffmann • \\ Kerry Dwan · Pierre-Alain Clavien · \\ Heiner C. Bucher
}

Published online: 9 September 2014

(C) Société Internationale de Chirurgie 2014

\begin{abstract}
Background Reporting of surgical outcomes is important for healthcare decision making and includes the reporting of complications. Several classifications have been proposed and validated for postoperative, but not intraoperative, complications. The aim of the present study is to assess the current practice of complication reporting in surgical trials.

Methods We evaluated the reporting of intra- and postoperative complications in all registered randomized controlled trials that included investigate surgery or invasive interventions in at least one study arm and were published in 2010 in the Annals of Surgery, JAMA Surgery, and the British Journal of Surgery.

Results A total of 46 trials were identified; intra- and postoperative complications were reported separately in $42 \%$ and pooled in $15 \%$. In $37 \%$ intraoperative, in $2 \%$ postoperative, and in $4 \%$ both intra- and postoperative, complications were not reported at all. Exact definitions were provided in $13 \%$ for intraoperative and in $50 \%$ for postoperative complications. A classification was used in $9 \%$ for intra- and in $54 \%$ for postoperative complications, most frequently according to severity. The type of intervention (surgical vs. other) or whether the primary outcome was the assessment of complications had no significant impact on reporting definitions of adverse events.

Conclusions Intraoperative complications are frequently pooled with postoperative complications, ill-defined, or not reported at all, hampering informed decision making. As further research, we propose to develop and validate a classification of intraoperative complications. This will facilitate the evaluation of safety and the continuous quality control of surgical interventions with the ultimate goal to contribute to patient safety.
\end{abstract}

Electronic supplementary material The online version of this article (doi:10.1007/s00268-014-2776-8) contains supplementary material, which is available to authorized users.

R. Rosenthal $(\bowtie) \cdot$ H. Hoffmann

Department of Surgery, University Hospital Basel, Spitalstrasse

26, 4031 Basel, Switzerland

e-mail: rachel.rosenthal@unibas.ch

H. Hoffmann

e-mail: henry.hoffmann@usb.ch

K. Dwan

Department of Biostatistics, The University of Liverpool,

Liverpool L69 3GS, UK

e-mail: Kerry.Dwan@liverpool.ac.uk
P.-A. Clavien

Department of Surgery, University Hospital Zurich, University of Zurich, Raemistrasse 100, 8091 Zurich, Switzerland e-mail: clavien@access.uzh.ch

H. C. Bucher

Basel Institute for Clinical Epidemiology and Biostatistics, University Hospital Basel, Hebelstrasse 10, 4031 Basel,

Switzerland

e-mail: heiner.bucher@usb.ch 


\section{Introduction}

High-level evidence is of utmost importance for decision making in healthcare. Therefore, reporting needs to be accurate and transparent. Reporting should follow standardized guidelines as proposed by CONSORT (Consolidated Standards of Reporting Trials Statement) [1]. Of specific interest to surgery are the CONSORT extensions for non-pharmacologic treatment [2]. There, challenges to surgical trials such as blinding, experience of and clustering by care providers and centers, and standardization of interventions are accounted for. The CONSORT statement was initially developed to improve reporting of efficacy of randomized controlled trials (RCTs). Since then, the group has published an extension for better reporting of harms in relation to interventions. There, it is specified that the methods section should include a list of addressed adverse events, with definitions for each and information on how harms-related data were collected [3]. If relevant, information on grading and expectancy of adverse events and validation of definitions should be provided. [3] In surgery, reporting of adverse events is specifically challenging due to the potential complexity of these events [2,4]. An analysis of the quality of reporting of short-term postoperative surgical complications after pancreatectomy, hepatectomy, and esophagectomy involving 119 articles, revealed that definitions of complications were provided in $34 \%$ of the articles and severity was graded in only $20 \%$ [4].

Whereas several simple and reproducible classification systems have been developed for postoperative complications [5-11], to our knowledge there is still no such classification specifically addressing intraoperative complications. To date, reporting of intraoperative complications in the literature is frequently either not present at all or intraoperative complications are pooled with postoperative complications [12]. If intraoperative complications are reported separately, they tend to be ill-defined or only roughly classified according to severity [13], for example, as 'minor' versus 'major', lacking exact classification criteria, and/or classified according to the injured organ (e.g. vascular, visceral, solid organ injury) without accounting for different degrees of severity [14]. A classification system of intraoperative complications is of particular interest and increasing importance in studies evaluating new surgical techniques and devices. It would allow for better transparency and more objective comparison between different surgical techniques and their outcomes. Such a system would inform a more differentiated judgment of the evidence and provide a better rationale for more individualized decision making in surgery. Additionally, standardization allows more adequate outcome reporting in educational and training settings (individual surgeon's outcome) and in institution benchmarking (institution outcome).
The aim of the proposed research is to systematically assess the current practice of reporting intra- and postoperative adverse events in RCTs published during 1 year in three major general surgery journals. This is of relevance to the subsequent development of a classification system for intraoperative complications.

\section{Materials and methods}

Included studies

This is a secondary analysis of a review investigating discrepancies between registry entries and final reports in three general (non-specialty) surgical journals [15]. The journals with the highest 2010 impact factor under the category 'surgery' (Annals of Surgery, JAMA Surgery [formerly Archives of Surgery], and British Journal of Surgery) were screened for all RCTs published between 1 January 2010 and 31 December 2010. Preclinical studies, duplicate publications, secondary analyses, interim reports, and trials lacking trial registry information (since in the primary review, registry entries were compared with final reports) were excluded. Since we considered all RCTs published in the above-mentioned journals, the RCTs were not limited to surgical or invasive treatments.

For the current investigation, trials not involving surgery or invasive treatments, such as trials investigating pharmacological interventions unrelated to surgery, or trials evaluating the informed consent process, were excluded since we could not explore the reporting of intraoperative complications in these trials.

Invasive interventions were defined as interventions requiring either percutaneous access (such as radiofrequency ablation of liver metastases) or access through natural orifices (such as endoscopic retrograde cholangiopancreatography, but not natural orifice transluminal endoscopic surgery). The study intervention was defined as perioperative if surgery was part of the study but the intervention in itself was not, such as peri- or intraoperative administration of a drug, nutrition, bowel preparation, or transplant organ preparation.

\section{Data extraction}

The three journals were screened for RCTs, and data concerning the study population, interventions, and outcomes were extracted as previously described [15]. The reviewers were not blinded for the names of the authors and journals of the evaluated RCTs. In the current investigation, two reviewers ( $\mathrm{RR}$ and $\mathrm{HH}$ ) independently additionally retrieved the following information concerning adverse events: whether the occurrence of adverse events was reported (or 
whether there was a statement that none occurred), whether adverse events were the primary outcome, whether both intraoperative/intra-interventional and postoperative/postinterventional adverse events were reported, and, if yes, separately or not. Moreover, the reviewers assessed whether a definition of adverse events was given in the methods section, whether a classification system was used, and, if yes, according to which specifier the events were classified. Adverse events were considered 'reported' if any deviation from the normal intra- or postoperative/post-interventional course (excluding sequelae and failures of cure) was reported or if it was reported that no such events occurred. Conversion, such as from laparoscopy to open surgery in itself was not considered a complication (i.e. it could be due to extensive tumor burden or adhesions), but conversion due to complications (e.g. bleeding) was considered a complication. Adverse events were classified as 'intraoperative' if they were reported to occur during the surgical or invasive intervention or 'postoperative' if they were reported to occur after the intervention.

Complications were considered to be 'completely defined' if either an exact definition was provided (such as for postoperative complications "any deviation from the normal postoperative course excluding sequelae and failures of cure" [7] ) or if a complete list of events considered as complications was provided. Complications were considered to be 'not completely defined' if examples, but not a complete list, of events considered to be complications were provided. If, in the methods section, it was mentioned that complications or the postoperative course were recorded without any further elaboration or if complications were listed in the results section only and not mentioned in the methods, complications were considered to be 'not defined'.

Complications were considered to be 'classified' if some type of classification was used, such as according to severity or according to affected organ, regardless of whether the classification had previously been validated and scored as 'not classified' if they were individually listed. Discrepancies between the two reviewers were resolved by discussion and agreement. For data extraction, an Excel spreadsheet (Microsoft Office XP, Microsoft Corporation, Redmond, WA, USA) was used.

\section{Statistical analysis}

Descriptive statistics were used to present the proportions of trials with definition of intraoperative and postoperative complications and with information concerning grading systems, if applicable (main analysis). We carried out two additional explorative analyses. We compared the definition of intra- and postoperative complications (1) in trials with surgical procedures in at least one study arm as the primary study intervention versus those with invasive interventions or perioperative interventions as study intervention; and (2) in trials with adverse events being the primary outcome and those with other types of primary outcomes. Fisher's exact test was used for the comparisons. The comparisons were post hoc analyses, explorative in nature, and without any correction for multiple testing. Trials not reporting intra- or postoperative complications at all were excluded for these two explorative analyses. Analyses were conducted using Intercooled Stata Version 12.1; StataCorp LP, College Station, TX, USA.

\section{Results}

\section{Characteristics of included studies}

Of 596 studies identified through the search of the three journals, 65 were retrieved in full text, while the remaining studies were not RCTs and were excluded (Fig. 1, adapted from Rosenthal and Dwan [15] ). Of these, a further 14 trials were excluded as they were interim reports $(n=1)$; secondary analyses $(n=5)$; not an RCT, in contrast to the information provided in the heading or abstract $(n=4)$; not registered RCTs (author information) $(n=2)$; or had no registration information $(n=2)$. Therefore, 51 studies were included (21 Annals of Surgery, four JAMA Surgery, and 26 British Journal of Surgery) [15]. The references of these 51 studies are given in the Electronic supplementary material 1 . For the current investigation, two studies investigating the informed consent process (References 10*, 24* in Electronic supplementary material 1) and three studies evaluating medical treatment options without any relation to surgery (References $43^{*}, 45^{*}$, $48^{*}$ in Electronic supplementary material 1 ) were additionally excluded, since we could not explore the reporting of intraoperative complications in these trials, leaving a total of 46 studies for this review. Of these, 20 studies (43\%) included surgery, five studies (11\%) an invasive intervention, and four studies $(9 \%)$ both surgery and an invasive intervention. In the remaining 17 studies (37\%), the study intervention consisted of perioperative interventions, but surgery was part of the study.

The baseline characteristics of all 46 studies with details on the population, the intervention, control, primary outcome, and result are presented in the Electronic supplementary material 2 .

Intraoperative and postoperative adverse event reporting (main analysis)

All 46 studies included some kind of adverse event reporting. The primary outcome was unrelated to adverse events in $67 \%(n=31)$, whereas the primary outcome was defined as the occurrence of any kind of adverse event in $33 \%(n=15)$. Of the latter, the adverse event related to 


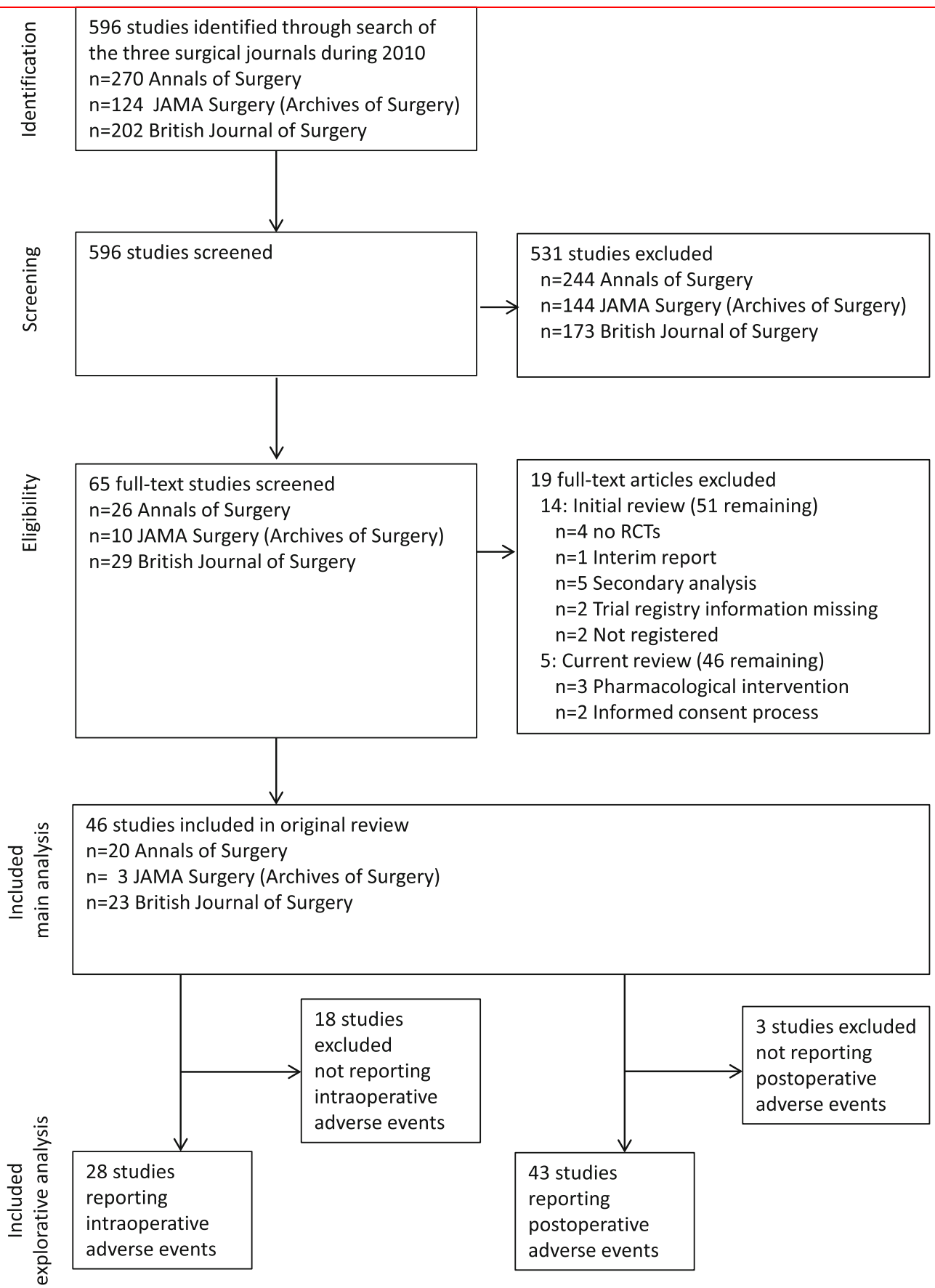

Fig. 1 Flow chart of included studies, adapted from Rosenthal and Dwan [15]. JAMA Journal of the American Medical Association, $R C T$ randomized controlled trial

postoperative complications in 14 studies, to intraoperative complications in none of the studies, and to adverse events unrelated to surgery in one study.

Intraoperative complications were reported separately from postoperative complications in $42 \%(n=19)$ and pooled in $15 \%(n=7)$, whereas in $37 \%(n=17)$ intraoperative, in $2 \%(n=1)$ postoperative, and in $4 \%$ $(n=2)$ both intra- and postoperative complications were not reported at all.

Intraoperative and postoperative complications were completely defined in $13 \%(n=6)$ and $50 \%(n=23)$, respectively; they were not completely defined in $4 \%$ $(n=2)$ and $2 \%(n=1)$, respectively; and were not defined at all in $44 \%(n=20)$ and $41 \%(n=19)$, 
Table 1 Definitions and classification of intra- and postoperative adverse events $(n=46)$

\begin{tabular}{lcc}
\hline & \multicolumn{2}{l}{ Period } \\
\cline { 2 - 3 } & Intraoperative & Postoperative \\
\hline Definition & & \\
Complete definition & $6(13)$ & $23(50)$ \\
No complete definition & $2(4)$ & $1(2)$ \\
No definition & $20(44)$ & $19(41)$ \\
No reporting of intra- respectively & $18(39)$ & $3(7)$ \\
$\quad$ postoperative AEs & & \\
Classification & & \\
Yes & $4(9)$ & $25(54)$ \\
No & $24(52)$ & $18(39)$ \\
No reporting of intra- respectively & $18(39)$ & $3(7)$ \\
$\quad$ postoperative AEs & & \\
Type of classification & & \\
Severity & $3(7)$ & $21(45)$ \\
Organ & $1(2)$ & $4(9)$ \\
No classification & $24(52)$ & $18(39)$ \\
No reporting of intra- respectively & $18(39)$ & $3(7)$ \\
$\quad$ postoperative AEs & & \\
\hline
\end{tabular}

Data are presented as $n(\%)$

AEs adverse events

respectively. A classification was used in $9 \%(n=4)$ for intraoperative and in $54 \%(n=25)$ for postoperative complications, most frequently according to severity (intraoperative complications: three of four, postoperative complications: 21 of 25, Table 1). Details on definition, classification, and occurrence of intraoperative and postoperative adverse events of the individual studies are presented in Electronic supplementary material 2. Of the 21 studies grading postoperative complications according to severity, seven used the generic classification of postoperative complications according to severity by Dindo et al. [7], taking into account the resulting type of complication management, e.g. the possibility of spontaneous resolution versus bedside procedure versus invasive procedure (Electronic supplementary material 2). One study referenced the generic classification used at the Memorial Sloan-Kettering Cancer Center [16]. Six studies used complication-specific classifications: three studies graded surgical site infections according to the definitions of the Centers for Disease Control and Prevention (Electronic supplementary material 2) $[17,18]$, one according to the ASEPSIS (Additional treatment, Serous discharge, Erythema, Purulent exudate, Separation of deep tissues) score [19], one graded complications after pancreatic surgery according to study group definitions [20-22], and one graded bleeding according to criteria defined in an RCT [23]. The remaining seven studies used their own definitions or did not provide any reference. As for the three studies grading intraoperative complications according to severity, one used the classification according to Dindo et al. [7], one derived the classification from a previous trial, and one used their own definition.

\section{Explorative analyses}

Three trials not reporting postoperative adverse events and 18 trials not reporting intraoperative adverse events were excluded.

Comparison of trials with surgical interventions versus those with invasive or perioperative interventions (explorative analysis 1)

A total of $46 \%(11 / 24)$ provided any type of definition of postoperative adverse events in trials with surgery as the study intervention versus $68 \%(13 / 19)$ in trials without surgery as the study intervention $(p=0.217)$.

In total, $18 \%(2 / 11)$ provided any type of definition of intraoperative complications in trials with surgical interventions versus $35 \%(6 / 17)$ in trials without surgical interventions $(p=0.419)$.

Comparison of trials with the primary outcome being adverse events versus not (explorative analysis 2)

A total of $71 \%(10 / 14)$ provided any type of definition of postoperative adverse events in trials where an adverse event was the primary outcome versus $48 \%(14 / 29)$ in trials with other types of primary outcomes $(p=0.199)$.

In total, $25 \%(2 / 8)$ provided any type of definition of intraoperative adverse events in trials where an adverse event was the primary outcome versus $30 \%(6 / 20)$ in trials with other types of primary outcomes $(p=1.000)$.

\section{Discussion}

This review of publications in three major surgical journals shows that intraoperative complications are rarely reported or they are subsumed with postoperative complications. If intraoperative complications are reported, they mostly lack a definition. This is in contrast to postoperative complications, for which about half of the studies provide these details. The type of study intervention or whether the primary outcome was an adverse event did not significantly impact on these results.

Relation to other studies

To our knowledge, this is the first review assessing the quality of reporting adverse events in surgery with a 
specific focus on intraoperative complications. However, the assessment of technical errors, mostly in the context of surgeon training and safety evaluation, has been previously investigated. As pointed out in the presidential address of the European Surgical Association by Clavien [24], targeting safety and quality in surgery including standardization of outcome measures is of utmost importance. In a review of studies describing technical errors during laparoscopy, Bonrath et al. [25] found 8 of 21 studies investigating surgical performance in terms of errors during routine laparoscopic cholecystectomy; in the remaining 13 studies, errors were evaluated as a surrogate for surgical skills in an educational context. Error definitions were found to vary considerably and hamper a direct comparison [25].

Several studies have investigated the reporting of postoperative surgical complications. A systematic review of the definition of anastomotic leak in gastrointestinal surgery based on 97 studies revealed 56 different definitions of anastomotic leak [26]. None of the studies used the standard definition for anastomotic leak that had previously been proposed at a consensus workshop [27]. Similarly, a systematic review of 90 studies on definitions of surgical wound infection revealed 41 different definitions. Of these, five may be considered as 'standard' definitions based on work by multidisciplinary groups [28]. Another review on the quality of surgery-related mortality reporting revealed considerable differences in follow-up time, and postdischarge mortality was rarely taken into account [29].

Thus, surgery-related complications and relevant outcomes in surgery lack standard definitions and therefore do not allow for comparison across studies and between surgical disciplines. However, this problem is not only related to surgery. Adverse event reporting in drug trials is also often lacking appropriate information about drug discontinuation and adequate reporting on toxicity and severity of adverse events [30, 31].

\section{Definition and classification of surgical complications}

Surgical complications are not uniformly defined. One option is to define surgical complications as "any undesirable, unintended, and direct result of an operation affecting the patient, which would not have occurred had the operation gone as well as could reasonably be hoped" [32]. This definition thus may apply to intra- and postoperative complications, but was regarded as controversial, because it may not always be possible to establish or rule out whether there is a causal relationship between surgery and the undesirable result. Moreover, not all undesirable results are complications, but they may also be inherent to the intervention or related to the severity of the underlying disease [33]. Thus, complications may be defined as "any deviation from the normal postoperative course" [7] and are distinguished from inherent effects of surgery (sequelae, such as the inability to walk after an amputation of a leg) or failures to cure (such as residual tumor after surgery) [5]. However, this definition is limited to postoperative complications. In a 5-year follow-up of the classification by Clavien et al. [6], the capturing of death during surgery or to "record as complication all events occurring in the operating room from the time of preparation for anesthesia" was discussed.

The need for a classification system of intraoperative complications

The need for an intraoperative classification of complications is subject to debate. Cunningham and Kavic [34] correctly point out that the definition of complications as deviation from the normal postoperative course [5, 7] does not capture intraoperative events that do not directly result in clinically relevant postoperative complications. They proposed to monitor any "deviation from the ideal operative course" and to distinguish between "simple errors" (adverse intraoperative events not leading to postoperative manifestations or additional risk for postoperative complications, e.g. burn to the lateral parietal peritoneum), and "complications" with potential risk for postoperative complications (e.g. inadvertent, but treated enterotomy or splenic injury) [34]. The concept was questioned by Wilson and Sokol [35], who argued that intraoperative complications that do result in a postoperative complication are captured as surgical complications anyway, whereas the others theoretically add information, but may complicate monitoring concepts. The authors propose to refer in such cases to "intraoperative errors", which of course need to be avoided.

Conversely, the documentation of intraoperative events may be especially important when new surgical techniques are introduced. In studies investigating new surgical procedures, the event rate of postoperative complications resulting from intraoperative complications may not be high enough to be captured with sample sizes resulting from calculations for efficacy outcomes. In contrast, documentation of intraoperative adverse events should prompt more extensive safety evaluation before wide application. The Global Harmonisation Task Force on Medical Devices (GHTF, see International Medical Device Regulators Forum IMDRF, www.imdrf.org) has provided guidance to standardize the reporting of adverse events and device failures [36]. In their guidelines, the GHTF study group distinguishes death, serious injury, and events 'potentially' leading to death or serious injuries, for instance, if they occurred again [37]. This concept is in line with a safety culture not limited to investigating actual patient injury in order to prevent reoccurrence of such an injury. Instead, such a concept also addresses critical incidents without 
actual patient injury and evaluates why such critical incidents and errors occur [38-40]. This is in accordance with the assumption that adverse events may arise from a buildup of several minor errors [41]. As a matter of fact, an error-outcome hierarchy may be described with the option of being uneventful on every hierarchy level [25, 42]. Starting with an error, such as inserting a trocar without visualization, a technical event such as an enteric injury may or may not occur. This injury may or may not be detected and rectified, leading or not to a postoperative complication such as an enteric leak. The latter in turn may or may not be detected and managed and, finally, may or may not result in an adverse outcome such as sepsis or death [42].

Additionally, some complications are just not captured when focusing only on the immediate postoperative phase, because the consequences are only evident in the long term or immediately and definitively treated during surgery, for instance, hemorrhage with intraoperative blood transfusion only.

\section{Limitations}

Our study presents some limitations. First, all RCTs published in three major surgical journals except those with strictly medical or informed consent process interventions were considered for review. Therefore, some of the interventions did not only involve surgery, but also invasive or perioperative interventions. We addressed this issue by evaluating, in an exploratory analysis, the impact of studies including surgery as the primary study intervention (yes vs. no) on complication reporting. Results from three journals may not be extrapolated to all surgical journals. We focused on three high-impact journals with an endorsed CONSORT reporting guidelines policy and it is therefore unlikely that an investigation in other surgical journals would yield lower frequencies of lack of definition and classification of intraand postoperative adverse effects. Second, reviewers were not blinded to authors and journals of the published RCTs. However data abstraction was carried out by two independent reviewers who used a standardized objective extraction protocol. We consider the lack of blinded data abstraction to be a minor risk of bias [43].

\section{Implications for daily practice and further research}

Reports on adverse events from surgical interventions often do not use uniform definitions for adverse events or do not distinguish between intra- and postoperative complications, and therefore have to be scrutinized. For these reasons, we plan to develop and validate a simple and reproducible classification for intraoperative complications, similar to the existing classifications of postoperative adverse events $[5-9,11]$.
The ultimate goal will be to propose a definition and classification of intraoperative complications that can be used for standardized outcome data collection. Therewith, a core outcome set that may be used as a reporting guideline for trials involving surgical procedures may be developed. This is in line with the COMET (Core Outcome Measures in Effectiveness Trials, www.comet-initiative. org) initiative, with the goal of developing reporting standards that allow for comparisons across studies, healthcare providers, and patients [44].

\section{Conclusions}

There is a lack of universal reporting of adverse events in surgery. This compromises the comparison of reports on intra- and postoperative complications in surgical innovation and clinical research as well as continuous quality assessment and benchmarking. Several straightforward postoperative complications classifications have been developed and validated, but intraoperative complications are frequently not reported, ill-defined, or pooled with postoperative complications. A clear distinction and classification of intraoperative complications is therefore needed to capture intraoperative events that eventually result in postoperative complications or increase the risk for such complications. This would also allow standardization of critical incident-reporting systems that may be needed for quality control, trainee assessments, or evaluation of surgeons' performance.

Acknowledgments There is no funding related to this publication. There are no conflicts of interest to declare. Heiner C. Bucher is supported by Grants from Santésuisse and the Gottfried and JuliaRhyner-Foundation. Rachel Rosenthal is an employee of F. Hoffmann-La Roche Ltd. since May 01, 2014. The present study was conducted before Rachel Rosenthal joined F. Hoffmann-La Roche Ltd. and has no connection to her employment by the company.

\section{References}

1. Schulz KF, Altman DG, Moher D (2010) CONSORT 2010 statement: updated guidelines for reporting parallel group randomised trials. BMJ 340:c332

2. Boutron I, Moher D, Altman DG, Schulz KF, Ravaud P (2008) Extending the CONSORT statement to randomized trials of nonpharmacologic treatment: explanation and elaboration. Ann Intern Med 148:295-309

3. Ioannidis JP, Evans SJ, Gotzsche PC, O'Neill RT, Altman DG, Schulz K, Moher D (2004) Better reporting of harms in randomized trials: an extension of the CONSORT statement. Ann Intern Med 141:781-788

4. Martin RC, Brennan MF, Jaques DP (2002) Quality of complication reporting in the surgical literature. Ann Surg 235:803-813

5. Clavien PA, Sanabria JR, Strasberg SM (1992) Proposed classification of complications of surgery with examples of utility in cholecystectomy. Surgery 111:518-526 
6. Clavien PA, Barkun J, de Oliveira ML, Vauthey JN, Dindo D, Schulick RD, de Santibañes E, Pekolj J, Slankamenac K, Bassi C, Graf R, Vonlanthen R, Padbury R, Cameron JL, Makuuchi M (2009) The Clavien-Dindo classification of surgical complications: five-year experience. Ann Surg 250:187-196

7. Dindo D, Demartines N, Clavien PA (2004) Classification of surgical complications: a new proposal with evaluation in a cohort of 6336 patients and results of a survey. Ann Surg 240:205-213

8. Pillai SB, van Rij AM, Williams S, Thomson IA, Putterill MJ, Greig S (1999) Complexity- and risk-adjusted model for measuring surgical outcome. Br J Surg 86:1567-1572

9. Pomposelli JJ, Gupta SK, Zacharoulis DC, Landa R, Miller A, Nanda R (1997) Surgical complication outcome (SCOUT) score: a new method to evaluate quality of care in vascular surgery. J Vasc Surg 25:1007-1014

10. Slankamenac K, Graf R, Barkun J, Puhan MA, Clavien PA (2013) The comprehensive complication index: a novel continuous scale to measure surgical morbidity. Ann Surg 258:1-7

11. Strasberg SM, Linehan DC, Hawkins WG (2009) The accordion severity grading system of surgical complications. Ann Surg 250:177-186

12. Tou S, Malik AI, Wexner SD, Nelson RL (2011) Energy source instruments for laparoscopic colectomy. Cochrane Database Syst $\operatorname{Rev}(5): C D 007886$

13. Morino M, Rimonda R, Allaix ME, Giraudo G, Garrone C (2005) Ultrasonic versus standard electric dissection in laparoscopic colorectal surgery: a prospective randomized clinical trial. Ann Surg 242:897-901

14. Ahmad G, O'Flynn H, Duffy JM, Phillips K, Watson A (2012) Laparoscopic entry techniques. Cochrane Database Syst Rev (2):CD006583

15. Rosenthal R, Dwan K (2013) Comparison of randomized controlled trial registry entries and content of reports in surgery journals. Ann Surg 257:1007-1015

16. Martin RC, Jaques DP, Brennan MF, Karpeh M (2002) Achieving RO resection for locally advanced gastric cancer: is it worth the risk of multiorgan resection? J Am Coll Surg 194:568-577

17. Horan TC, Gaynes RP, Martone WJ, Jarvis WR, Emori TG (1992) CDC definitions of nosocomial surgical site infections, 1992: a modification of CDC definitions of surgical wound infections. Infect Control Hosp Epidemiol 13:606-608

18. Mangram AJ, Horan TC, Pearson ML, Silver LC, Jarvis WR (1999) Guideline for prevention of surgical site infection, 1999. Hospital Infection Control Practices Advisory Committee. Infect Control Hosp Epidemiol 20:250-278

19. Wilson AP, Treasure T, Sturridge MF, Gruneberg RN (1986) A scoring method (ASEPSIS) for postoperative wound infections for use in clinical trials of antibiotic prophylaxis. Lancet 1:311-313

20. Bassi C, Dervenis C, Butturini G, Fingerhut A, Yeo C, Izbicki J, Neoptolemos J, Sarr M, Traverso W, Buchler M (2005) Postoperative pancreatic fistula: an international study group (ISGPF) definition. Surgery 138:8-13

21. Wente MN, Bassi C, Dervenis C, Fingerhut A, Gouma DJ, Izbicki JR, Neoptolemos JP, Padbury RT, Sarr MG, Traverso LW, Yeo CJ, Buchler MW (2007) Delayed gastric emptying (DGE) after pancreatic surgery: a suggested definition by the International Study Group of Pancreatic Surgery (ISGPS). Surgery 142:761-768

22. Wente MN, Veit JA, Bassi C, Dervenis C, Fingerhut A, Gouma DJ, Izbicki JR, Neoptolemos JP, Padbury RT, Sarr MG, Yeo CJ, Buchler MW (2007) Postpancreatectomy hemorrhage (PPH): an International Study Group of Pancreatic Surgery (ISGPS) definition. Surgery 142:20-25
23. Fox KA, Mehta SR, Peters R, Zhao F, Lakkis N, Gersh BJ, Yusuf S (2004) Benefits and risks of the combination of clopidogrel and aspirin in patients undergoing surgical revascularization for nonST-elevation acute coronary syndrome: the Clopidogrel in Unstable angina to prevent Recurrent ischemic Events (CURE) Trial. Circulation 110:1202-1208

24. Clavien PA (2013) Targeting quality in surgery. Ann Surg 258:659-668

25. Bonrath EM, Dedy NJ, Zevin B, Grantcharov TP (2013) Defining technical errors in laparoscopic surgery: a systematic review. Surg Endosc 27:2678-2691

26. Bruce J, Krukowski ZH, Al-Khairy G, Russell EM, Park KG (2001) Systematic review of the definition and measurement of anastomotic leak after gastrointestinal surgery. $\mathrm{Br}$ J Surg 88:1157-1168

27. Peel AL, Taylor EW (1991) Proposed definitions for the audit of postoperative infection: a discussion paper. Surgical Infection Study Group. Ann R Coll Surg Engl 73:385-388

28. Bruce J, Russell EM, Mollison J, Krukowski ZH (2001) The quality of measurement of surgical wound infection as the basis for monitoring: a systematic review. J Hosp Infect 49:99-108

29. Russell EM, Bruce J, Krukowski ZH (2003) Systematic review of the quality of surgical mortality monitoring. Br J Surg 90:527-532

30. Ioannidis JP, Contopoulos-Ioannidis DG (1998) Reporting of safety data from randomised trials. Lancet 352:1752-1753

31. Ioannidis JP, Lau J (2001) Completeness of safety reporting in randomized trials: an evaluation of 7 medical areas. JAMA 285:437-443

32. Sokol DK, Wilson J (2008) What is a surgical complication? World J Surg 32:942-944

33. Dindo D, Clavien PA (2008) What is a surgical complication? World J Surg 32:939-941

34. Cunningham SC, Kavic SM (2009) What is a surgical complication? World J Surg 33:1099-1100

35. Wilson J, Sokol DK (2009) Do we need a concept of intraoperative complication? World J Surg 33:1102

36. Mehran R, Leon MB, Feigal DA, Jefferys D, Simons M, Chronos N, Fogarty TJ, Kuntz RE, Baim DS, Kaplan AV (2004) Postmarket approval surveillance: a call for a more integrated and comprehensive approach. Circulation 109:3073-3077

37. International Medical Device Regulators Forum (2006) Medical Devices Post Market Surveillance: Global Guidance for Adverse Event Reporting for Medical Devices. http://www.imdrf.org/ docs/ghtf/final/sg2/technical-docs/ghtf-sg2-n54r8-guidanceadverse-events-061130.pdf. Accessed 23 Oct 2013

38. Kram R (2008) Critical incident reporting system in emergency medicine. Curr Opin Anaesthesiol 21:240-244

39. Leape LL (1994) Error in medicine. JAMA 272:1851-1857

40. Reason J (2000) Human error: models and management. BMJ 320:768-770

41. Catchpole K (2010) Errors in the operating theatre-how to spot and stop them. J Health Serv Res Policy 15(Suppl 1):48-51

42. Bonrath EM, Zevin B, Dedy NJ, Grantcharov TP (2013) Error rating tool to identify and analyse technical errors and events in laparoscopic surgery. Br J Surg 100:1080-1088

43. Morissette K, Tricco AC, Horsley T, Chen MH, Moher D (2011) Blinded versus unblinded assessments of risk of bias in studies included in a systematic review. Cochrane Database Syst Rev (9):MR000025

44. Williamson PR, Altman DG, Blazeby JM, Clarke M, Devane D, Gargon E, Tugwell P (2012) Developing core outcome sets for clinical trials: issues to consider. Trials 13:132 